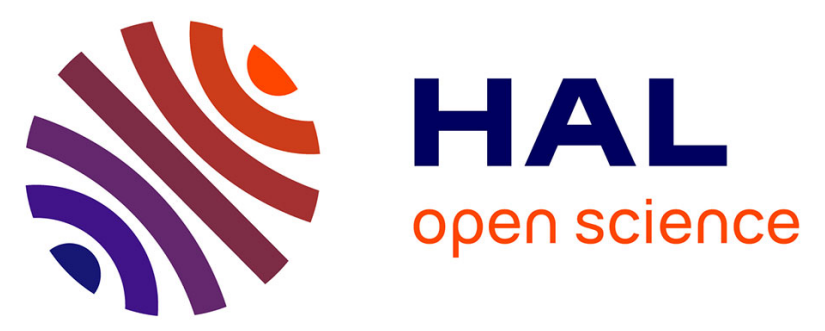

\title{
Matching of fundamental modes at a junction of a cylinder and a truncated cone; application to the calculation of some radiation impedances
}

\author{
Jean Kergomard, Antoine Lefebvre, Gary Scavone
}

\section{- To cite this version:}

Jean Kergomard, Antoine Lefebvre, Gary Scavone. Matching of fundamental modes at a junction of a cylinder and a truncated cone; application to the calculation of some radiation impedances. Acta Acustica united with Acustica, 2015, 101 (6), pp.1189-1198. 10.3813/AAA.918912 . hal-01134302v2

HAL Id: hal-01134302

https://hal.science/hal-01134302v2

Submitted on 28 Apr 2016

HAL is a multi-disciplinary open access archive for the deposit and dissemination of scientific research documents, whether they are published or not. The documents may come from teaching and research institutions in France or abroad, or from public or private research centers.
L'archive ouverte pluridisciplinaire HAL, est destinée au dépôt et à la diffusion de documents scientifiques de niveau recherche, publiés ou non, émanant des établissements d'enseignement et de recherche français ou étrangers, des laboratoires publics ou privés. 


\title{
Matching of fundamental modes at a junction of a cylinder and a truncated cone; application to the calculation of some radiation impedances
}

\author{
Jean Kergomard * \\ LMA, CNRS, UPR 7051, Aix-Marseille Univ, Centrale Marseille, \\ F-13402 Marseille Cedex 20, France \\ Antoine Lefebvre and Gary P. Scavone \\ Computational Acoustic Modeling Laboratory, \\ Centre for Interdisciplinary Research in Music Media and Technology (CIRMM \\ Schulich School of Music, \\ McGill University, \\ 555 Sherbrooke Street West, Montréal, Québec H3A 1E3, Canada
}

September 10, 2015

\begin{abstract}
The problem of the junction between a cylinder and a truncated cone at frequencies below the first cutoff of the cylinder is investigated, in particular for the case of acute angles. An analytical model of the matching of a cylinder and a truncated cone is derived for the general case of a cone of finite length having a known terminal impedance. When the cone is infinite and the angle is right, the problem is similar to the classical problem of a tube radiating in an infinite baffle. The model is based on a general formulation of the junction of several waveguides at low frequencies (when only the fundamental mode propagates in each guide), and on the assumption that at high frequencies, the radiation impedance of the cylinder is equal to its characteristic
\end{abstract}

*Tel 33 491164381, Fax 33 491228248, kergomard@lma.cnrs-mrs.fr 
impedance. The model has the form of an equivalent circuit, and involves several parameters related to the geometry (the areas of the surfaces defining the matching cavity and the volume of this cavity). In addition, the model requires one supplementary parameter only, i.e., the zero frequency value of the added mass (or length correction), which has to be determined numerically (the Finite Element Method is used). Analytical and numerical results agree very well at low and moderate frequencies, up to the cutoff of the first higher-order mode. For the radiation into an infinite flange, the results improve upon those in a recent publication that were obtained by optimization. The case of obtuse angles is more complicated and is briefly discussed. Finally for the case of infinite cones, the reflection coefficient is compared to that obtained in previous studies.

keywords: conical tubes, acoustic radiation impedance, cylinder-cone, wind instruments

\section{Introduction}

The calculation of the radiation of tubes into infinite space, when only the fundamental, planar mode propagates, is a classical issue: the definitive papers are by Levine and Schwinger [1] for a tube without flange and Norris and Sheng [2] for a tube in an infinite flange. Conversely the more general problem of the radiation of a cylindrical tube into a conical tube has been rarely treated using analytical methods, but Chester [3] and Martin [4] gave interesting formulas for the case of small angles. The present paper investigates this problem, but generalizes it by considering the possibility of a finite length for the truncated cone. In other words, the matching of planar waves in a cylinder and spherical waves in a truncated cone is studied. Radiation will be a particular case when the cone is infinite. The basis of the study is a general formulation for the junction of several waveguides at low frequencies [5], i.e., when only the fundamental mode propagates in each guide.

Figure 1 shows the geometry of the problem. Both tubes are without mean flow and with perfectly rigid walls: thus the fundamental modes are uniform modes in both tubes, i.e. their amplitude is constant in the transverse dimensions of the guides (on a plane for the cylindrical tube; on a spherical cap for the conical one). The subscript 0 refers to the output of the cylinder, while the subscripts 1 and 2 correspond to the input and output of the truncated cone. $\vartheta$ is the half-angle at the cone apex. 


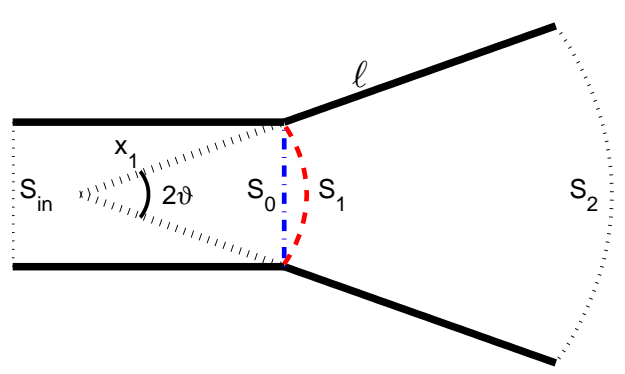

Figure 1: Geometry of the problem. The total length of the cone is $x_{2}=$ $\ell+x_{1}$, the half-angle at the apex is $\vartheta$. The plane surface, $S_{0}=\pi^{2} r_{0}$ (dasheddotted line) and the spherical cap $S_{1}=2 \pi x_{1}^{2}(1-\cos \vartheta)$ (dashed line) define the junction of the two waveguides.

Section 2 describes the transfer matrix for the fundamental mode of spherical waves in a truncated cone. Section 3 presents the problem as a junction of two waveguides and the formulation as an equivalent electric circuit. Section 4 shows that this formulation, together with an assumption concerning the high frequency behavior, leads to a general approximate formula for the output impedance of the cylinder. With the knowledge of the geometry and only one supplementary parameter, which is the added mass at zero frequency, it is possible to deduce a complete impedance curve at frequencies lying below the cutoff of the first-order higher mode in the cylinder. This is the main result of the present paper. For the particular case of an infinite cone and an angle $\vartheta=\pi / 2$, this formula is compared with the exact result given by Norris and Sheng [2].

For other values of $\vartheta$, no exact result is available. The Finite Element Method (FEM) is used for the case of cones of finite length, and this allows the added mass to be determined and then the comparison of the analytical formula with the numerical result. Section 6 presents the problem of obtuse angles. Finally, Section 7 presents the results of Chester [3] and Martin [4] in comparison with those given by the present method, for the case of an infinite cone. 


\section{Input admittance of a conical tube}

Using a change in variables, the propagation of the fundamental mode of spherical waves can be classically described by a transfer matrix identical to that of planar waves (see e.g. [6]), as follows:

$$
\begin{aligned}
\left(\begin{array}{c}
P_{1} \\
\widetilde{V}_{1}
\end{array}\right) & =\frac{x_{2}}{x_{1}}\left(\begin{array}{cc}
\cos k \ell & j \rho c \sin k \ell \\
j(\rho c)^{-1} \sin k \ell & \cos k \ell
\end{array}\right)\left(\begin{array}{c}
P_{2} \\
\widetilde{V}_{2}
\end{array}\right) \\
\text { with } \tilde{V}_{i} & =V_{i}-\frac{P_{i}}{j k \rho c x_{i}} \quad i=1,2
\end{aligned}
$$

The equation is written in the frequency domain. $P_{i}$ and $V_{i}$ are the acoustic pressure and velocity at the distance $x_{i}$ from the apex, $j^{2}=-1$ (the assumed time dependence is $\exp (j \omega t)), c$ the speed of sound, $\rho$ the gas density $k=\omega / c, \omega$ the angular frequency, $\ell=x_{2}-x_{1}$. The quantity $\widetilde{V}_{i}$ can be called the symmetric velocity, because the asymmetric transmission line corresponding to spherical waves becomes symmetrical with this appropriate choice of variables. This allows a symmetric acoustic admittance, $\widetilde{Y}_{i}$ to be defined as:

$$
\widetilde{Y}_{i}=\frac{S_{i} \widetilde{V}_{i}}{P_{i}}=Y_{i}-\frac{S_{i}}{j \omega \rho x_{i}}
$$

where $S_{i}$ is the area of a spherical cap at distance $x_{i}$ from the apex, which is equal to $S_{i}=2 \pi x_{i}^{2}(1-\cos \vartheta)$, with $\sin \vartheta=r_{0} / x_{1}$.

The fundamental mode is the only propagating mode up to the cutoff frequency of the first higher-order mode. In Ref. [7], an approximate formula is given for this frequency, which depends on the abscissa in the cone (thus on the radius $r$ at this abscissa). We write it in the following form:

$$
k r=\sqrt{\mu(\mu+1)} \sin \vartheta \text { with } \mu=\frac{3.832}{\vartheta(1+0.14 \vartheta)} .
$$

When $\vartheta$ tends to 0 (case of a cylinder), $k r$ tends to the well known value of 3.832 for a cylindrical tube. For $\vartheta$ increasing toward $\pi / 2$, the value of $k r$ decreases monotonically to $\sqrt{6}=2.45(\mu=2)$.

\section{Matching of a cylinder and a truncated cone}

In Ref. [5], a general formulation for the junction of several cylindrical guides was proposed. The formulation takes into account the existence of evanescent modes in the different guides and allows matching the propagating modes. For the junction of two guides (with indices 0 and 1), either cylindrical or 
conical, with only one mode propagating in each, the matching of the propagating modes in the two guides reduces to (see Appendix A for the derivation):

$$
\left(\begin{array}{l}
P_{0} \\
P_{1}
\end{array}\right)=\left[\frac{\rho c^{2}}{j \omega V}\left(\begin{array}{ll}
1 & 1 \\
1 & 1
\end{array}\right)+j \omega\left(\begin{array}{ll}
M_{00} & M_{01} \\
M_{10} & M_{11}
\end{array}\right)+O\left(\omega^{3}\right)\right]\left(\begin{array}{c}
U_{0} \\
-U_{1}
\end{array}\right)
$$

$P_{0,1}$ and $U_{0,1}$ are the acoustic pressure and flow rate of the fundamental mode, respectively. The subscripts 0 and 1 are related to the surfaces defining the junction cavity: these surfaces can be chosen arbitrarily, but it is convenient to use a cavity as small as possible. The shape of the first matrix is due to the mass conservation ( $V$ is the volume of the junction). The minus sign before $U_{1}$ is used for convenience for ensuring the symmetry of the input and output, thus for exhibiting the symmetry of the matrix $\mathbf{M}$ (which is due to reciprocity). In this paper, bold characters are used for vectors and matrices. $\mathbf{M}$ is proportional to the density $\rho$. The elements $M_{i j}$, which represent the effect of evanescent modes on both sides of the junction, can be calculated by solving the Laplace equation (for an incompressible fluid). The terms of higher order in frequency $\left(\omega^{3}\right)$ are matrices depending on both the compressibility and the density. Equivalently, they could be replaced by a variation of the elements of the matrix $\mathbf{M}$ with frequency, writing a quantity like $M+\omega^{2} M^{\prime}$ as $M(\omega)$. However the present paper is limited to the first order.

Equation (4) is assumed to be valid below the cutoff frequencies of the first higher order mode in each guide. The equivalent electrical circuit is shown in Fig. 2, The result is formal, because the method is in general not an effective computation method of the elements of the matrices.

The matching volume between the output of the cylinder and the input of the truncated cone (see Fig. 1) has the following expression:

$$
V=\pi r_{0}^{3} \frac{\sin \vartheta[2+\cos \vartheta)]}{3[1+\cos \vartheta]^{2}} \text {. }
$$

It is convenient to reduce the problem by using dimensionless variables: the impedances are reduced by the characteristic impedance of the cylindrical tube, $Z_{c 0}=\rho c /\left(\pi r_{0}^{2}\right)$. Lowercase characters are used for the dimensionless variables (e.g. $y_{1}$ is the dimensionless version of $Y_{1}$ in Eq. (2)). $k r_{0}$ is the reduced frequency, and the Laplace variable is noted $s=j k r_{0}$. The following 


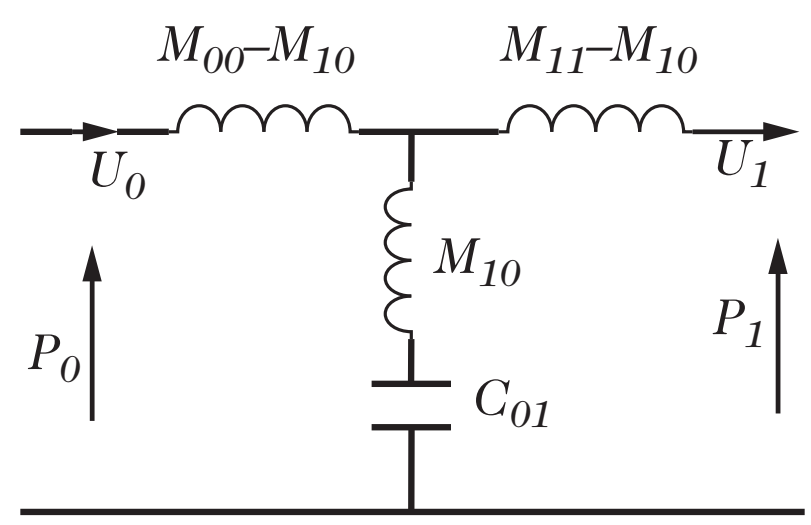

Figure 2: Equivalent electrical circuit of the junction between the two guides. The subscripts 0 and 1 correspond to the cylindrical and conical guides, respectively. $C_{01}=V / \rho c^{2}$, where $V$ is the volume of the junction.

expressions are found:

$$
\begin{aligned}
y_{1}= & \widetilde{y}_{1}+\frac{1}{s m_{1}}, \text { with } m_{1}=\frac{\zeta}{\sin \vartheta} ; \zeta=\frac{1+\cos \vartheta}{2} ; \\
& j \omega \frac{V}{\rho c^{2}} \frac{\rho c}{\pi r_{1}^{2}}=s c_{01} \quad \text { with } c_{01}=\frac{V}{\pi r_{0}^{3}} ; \\
j \omega M_{i j} \frac{\pi r_{1}^{2}}{\rho c}= & s m_{i j}, \text { where } m_{i j}=M_{i j} \frac{\pi r_{0}}{\rho}
\end{aligned}
$$

$\zeta$ is the ratio of plane to spherical surfaces. The output impedance $z_{0}=$ $P_{0} / U_{0}$ of the cylindrical tube is given by Eq. (4), or by the equivalent circuit of Fig. 2. After some algebra, it is found to be:

$$
z_{0}=\frac{\left[\frac{1}{y_{1}}+s m_{11}-s m_{10}\right]\left[\frac{1}{s c_{01}}+s m_{10}\right]}{\left[\frac{1}{s c_{01}}+\frac{1}{y_{1}}+s m_{11}\right]}+s\left(m_{00}-m_{10}\right) .
$$

\section{General formulas for the output impedance of the cylinder}

The above formula is valid whatever the output admittance of the cone at the surface $S_{2}$. However some knowledge concerning the particular case of infinite cones will be useful for determining the masses $m_{i j}$. 


\subsection{Conditions for the case of an infinite conical tube}

Consider an infinite conical tube. It is assumed that for any value of $\vartheta$, the high-frequency asymptotic value of a radiation impedance $z_{0}$ is the characteristic impedance $Z_{c 0}$ of the fundamental mode in the cylinder. Relationships between the masses $m_{i j}$ are sought by using this assumption. At the input of the truncated cone, the symmetric admittance is $\widetilde{Y}_{1}=S_{1} / \rho c$, thus:

$$
y_{1}=\frac{1}{\zeta}+\frac{1}{s m_{1}}=\frac{1}{\zeta}\left[1+\frac{\sin \vartheta}{s}\right] .
$$

At higher frequencies, the compliance term $1 /\left(s c_{01}\right)$ is of order $s^{-1}$, and can be neglected in Eq. (9). Similarly $1 / y_{1} \simeq \zeta$. Thus at higher frequencies:

$$
z_{0} \simeq \frac{-s^{2} m_{10}^{2}}{\zeta+s m_{11}}+s m_{00}=1
$$

The identification of the two highest order terms leads to:

$$
\begin{aligned}
& m_{10}^{2}=m_{11} m_{00} \\
& m_{11}=\zeta m_{00} .
\end{aligned}
$$

Eq. (12) gives two solutions for $m_{10}$. The positive value leads to a resonant curve for the output impedance, while the negative value gives a very good approximation formula, as shown hereafter.

When the frequency tends to 0, the series expansion of Eq. (9) with Eq. (10) is done at the first order of $s . z_{0}$ reduces to the impedance of a mass, and is denoted $s m$ :

$$
z_{0}=s m=s\left(m_{1}+m_{11}-2 m_{10}+m_{00}\right) .
$$

$m$ needs to be computed by numerical methods (which is done in Section 5). It is the coefficient of the so-called zero frequency "length correction" at the end of the cylindrical tube, or more accurately, the zero frequency added mass. For the limit case $\vartheta=\pi / 2$ (tube in an infinite baffle), it is known to be $m=0.82159$ (see [2,8]). With the knowledge of this quantity, the model is complete. The three masses $m_{i j}$ are given by:

$$
\begin{aligned}
& m_{00}=\left(m-m_{1}\right) /(1+\sqrt{\zeta})^{2} \\
& m_{11}=\zeta m_{00} ; m_{10}=-\sqrt{\zeta} m_{00}
\end{aligned}
$$




\subsection{General and simplified formulas}

With the expressions $(15)$ and $(16)$, the general formula is obtained from Eq. (9), as follows:

$$
z_{0}=\frac{1+y_{1} s\left(m-m_{1}\right)+s^{2} c_{01} m_{00}}{s c_{01}+y_{1}\left(1+s^{2} c_{01} m_{11}\right)} \text {. }
$$

For the case of an infinite cone, this formula becomes:

$$
z_{0}=\frac{s m+s^{2}\left(m-m_{1}\right) m_{1} / \zeta+s^{3} m_{1} c_{01} m_{00}}{1+s m_{1} / \zeta+s^{2} c_{01}\left(m_{11}+m_{1}\right)+s^{3} m_{1} c_{01} m_{00}} .
$$

When the frequency tends to 0 , it can be checked that:

$$
\operatorname{Re}\left(z_{0}\right)=-s^{2} m_{1}^{2} / \zeta=\operatorname{Re}\left(1 / y_{1}\right) .
$$

This is due to the conservation of both the acoustic power and the flow rate between the two surfaces $S_{0}$ and $S_{1}$.

In Section 5 it will be shown that $m-m_{1} \approx \zeta c_{01}$. Thus a simplified expression can be found for Eq. (17), in particular for smaller angles and lower frequencies:

$$
z_{0}=\frac{1+y_{1} s c_{01} \zeta}{s c_{01}+y_{1}}
$$

\subsection{Radiation into an infinite flange}

For the case $\vartheta=\pi / 2$, Eq. (18) becomes:

$$
z_{0}=\frac{0.82159 s+0.3216 s^{2}+0.0368 s^{3}}{1+s+0.3701 s^{2}+0.0368 s^{3}}
$$

For this case, looking at the poles of the denominator, it can be checked that two roots are complex conjugate and one is real, all real parts being negative. Thus the inverse Fourier Transform is causal. Figure 3 shows the comparison of the exact result (Ref. [2]) with the approximate formula given in Ref. [9], and with Eq. (21). Notice that the aim of Ref. [9] was to propose approximate formulas satisfying Hermitian symmetry for the reflection coefficient, and causality for the impulse response. These two requirements are satisfied by Eq. 21). Except at very high frequencies, this formula is excellent. This is remarkable, because it is built with the knowledge of the zero frequency added mass only. As explained in Section 2, the expected limit of validity of Eq. (21) is $k r_{0}=2.45$. However the equation remains valid for frequencies above this limit, where at least two propagating modes are expected, as well as a corresponding directivity pattern in the radiated field. 


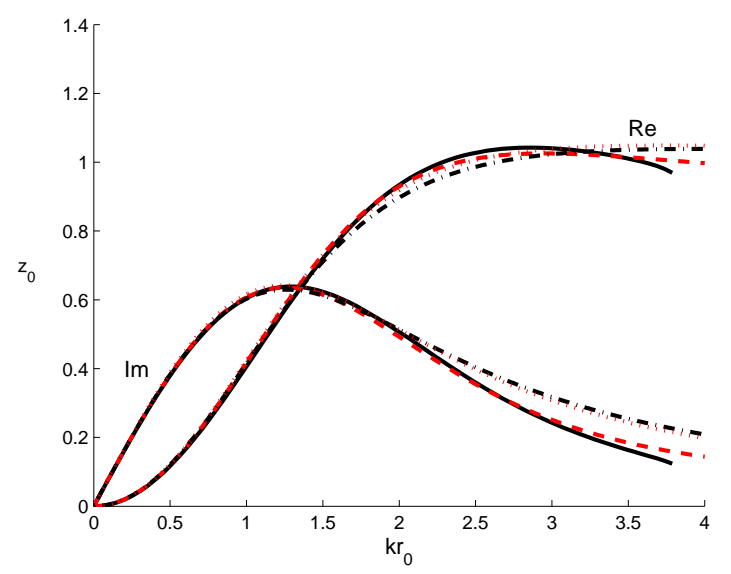

Figure 3: Real and imaginary parts of the radiation impedance $z_{0}$ in an infinite flange $(\vartheta=90)$. Black, solid line: exact result [2]; black, dash-dot line, approximate result [9]; grey (red online), dashed line: Eq. (21); grey (red online), dotted line: Eq. (22).

For the same case, $c_{01}=2 / 3$, and Eq. (20) becomes:

$$
z_{0}=\frac{5 s / 6+s^{2} / 3}{1+s+s^{2} / 3} \text {. }
$$

The simplicity of this formula is remarkable. $5 / 6=0.8333$ is very close to the exact value of the length correction at zero frequency. The other coefficients are close to those obtained by optimization in [9]: 0.324 instead of $1 / 3$ and 1.003 instead of 1 (for the term in $s$ ) in the denominator. Figure 3 shows that the result of Eq. (22) is very close to that of Ref. [9].

\section{Numerical calculation of the zero frequency added mass}

\subsection{Method}

For $\vartheta<\pi / 2$, the added mass coefficient $m$ is not known. Therefore the Finite Element Method is used. In order to avoid complications with an anechoic termination, two reactive boundary conditions are investigated: i) a rigid wall; and ii) a perfectly soft wall. In both cases the wall is perfectly spherical and the condition is valid at every angle, thus at the input of the cone, ideally only the fundamental mode can be propagating under the cutoff 
of the higher-order modes. The cylinder has a certain length, $\ell_{0}$, sufficient to avoid the presence of evanescent modes at its input (at surface $S_{i n}$ ). The boundary value problem is as follows: the Helmholtz equation is applied to the volume comprised between surfaces $S_{i n}$ and $S_{2}$, and the other walls are defined as rigid (Neumann boundary condition). The spherical boundary at the output of the cone was defined as either rigid or soft $\left(P_{2}=0\right)$. On the surface $S_{\text {in }}$ a unit acceleration is fixed at a constant value and the pressure is computed on the surface $S_{i n}$. Thus the input impedance is computed on the same surface, then it is projected to the output of the cylinder, giving the desired impedance $z_{0}$ on surface $S_{0}$, and the mass $m$ is obtained by using formula (17) in the limit of zero frequency. The choice of solving the Helmholtz equation is done in order to compare the numerical results with the results of the analytical formulas, at non zero frequencies (see Section 5.3 .

The FE model was formed as an axi-symmetric geometry. The cylinder radius and length were 1 and $5 \mathrm{~cm}$, respectively, and the truncated conic section was $5 \mathrm{~cm}$ long, as measured along its central axis, for all cone half angles. A fine mesh was constructed with triangular elements and a maximum element size of 0.001 meter. Numerical simulations were conducted for cone half angles from 5 to 105 degrees in increments of 5 degrees, with an additional set of simulations at 115, 125, and 135 degrees.

\subsection{Numerical result for the zero frequency added mass}

From the knowledge of the admittance at the output of the cone, $Y_{2}$, the input admittance $y_{1}$ is derived. Using Eqs. (1) and (6), the following expression is found:

$$
y_{1}=\frac{1}{s m_{1}}+\frac{1}{\zeta} \frac{j \tan k \ell+\widetilde{Y}_{2} \rho c / S_{2}}{1+j \widetilde{Y}_{2} \rho c / S_{2} \tan k \ell} .
$$

For a perfectly soft termination $\left(Y_{2}=\infty\right), \widetilde{Y}_{2}$ is infinite, while for a perfectly rigid termination, $\widetilde{Y}_{2} \rho c / S_{2}=-1 /\left(j k x_{2}\right)$. In both cases, the admittance $y_{1}$ is purely imaginary. The value of the mass $m$ is derived from Eq. (17):

$$
m=m_{1}+\frac{z_{0}\left(y_{1}+s c_{01}\right)-1}{s\left[y_{1}+s c_{01}\left(1-\zeta z_{0} y_{1}\right) /(1+\sqrt{\zeta})^{2}\right]} .
$$

For $k r_{0}=0.01$, the accuracy of the value of $m$ can be assessed to be better than $0.1 \%$, when changing the frequency to another very low frequency, or when changing the termination (soft to rigid), or when changing the length $\ell$ of the truncated cone. The calculation is done for several values of $\vartheta$. Figure 


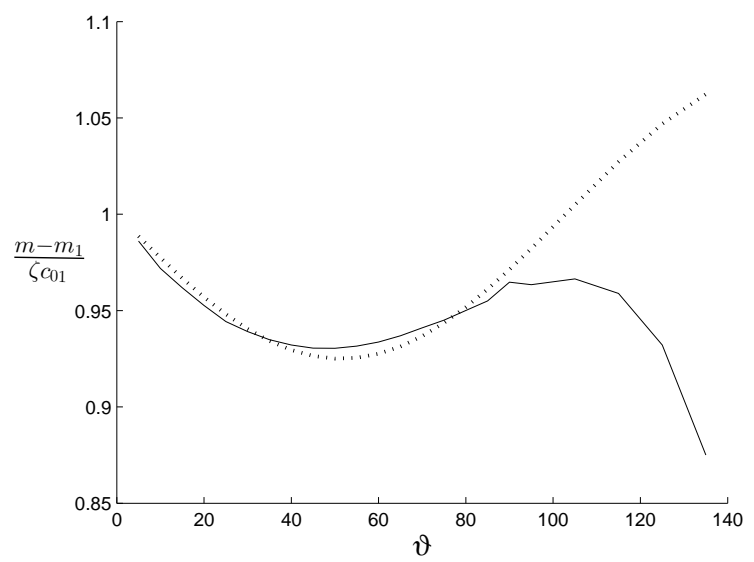

Figure 4: The quantity $(m-m 1) / \zeta c_{10}$ with respect to the angle $\vartheta$ (in degrees). Dotted line: expression given by Eq. 225.

4 shows that the mass $m-m_{1}$ is roughly proportional to the quantity $\zeta c_{01}$, the ratio ranging between 0.93 and unity. A fit formula is found to be:

$$
m-m_{1} \simeq \zeta c_{01}[1-0.075 \sin (1.75 * \vartheta)]
$$

with an error smaller than $1 \%$ for $\vartheta<\pi / 2$. For small angles, $\zeta c_{01} \ll m_{1}$. This leads to the simplest value for $m: m=m 1=0.5 \cot (\vartheta / 2)$. The latter expression is equivalent to that of Tyte [10].

\subsection{Verification for the case of cones of finite length}

With the value obtained for the mass $m$, it is possible to compare the general model (17) with the FEM result for a cone of finite length and for the two terminations considered. The model is found to be very good, as shown in Figs. 5 and 8 for the case $\vartheta=50$. For this angle, the cutoff frequency of the first higher order mode at the cone input is $k r_{0}=3.36$. Figure 6 shows the pressure field for a frequency higher than the cutoff $\left(k r_{0}=3.57\right)$. A slight dependence of the field on the angle $\vartheta$ is observed.

At rather high frequencies, the FEM results exhibit thin peaks on the output impedance curve: these peaks do not exist in the analytical formula. The reason lies in the creation of higher order spherical, propagating modes. The above mentioned cutoff value $k r_{0}=3.57$ corresponds to the property of the modes at the entry of the truncated cone. However, the duct modes which are evanescent at this location are propagating far from the entry. For the example computed, the cutoff at the output of the truncated cone is 


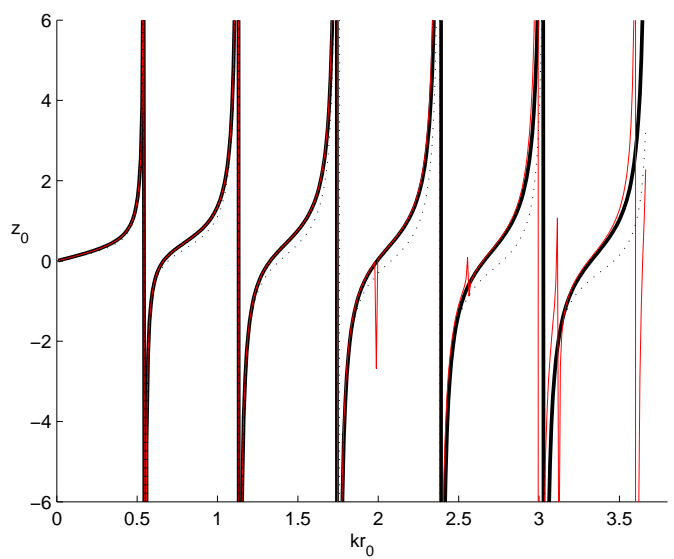

Figure 5: Output impedance $z_{0}$ (imaginary part) of a cylinder terminated in a truncated cone, the latter being terminated into a perfectly soft spherical cap. $\vartheta=50$. FEM result: grey (red online) line; Formula (17): black, solid line; Formula (20): dotted line. At low frequencies, the FEM results and Eq. (17) are indistinguishable. Above middle frequencies, non-axisymmetric modes appear in the FEM curve.
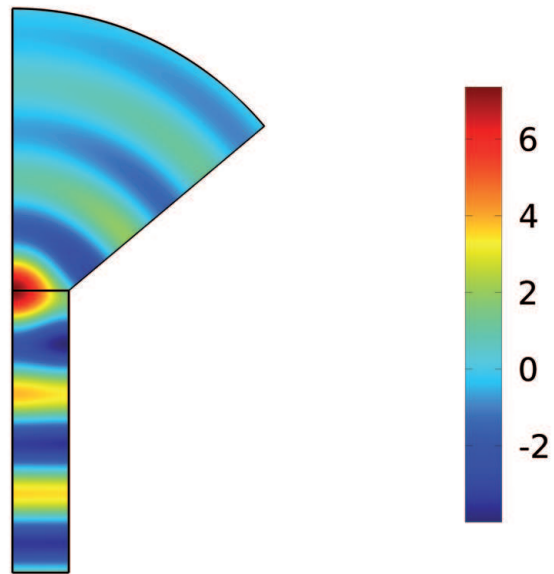

Figure 6: Pressure field for a frequency above the first cutoff frequency: $k r_{0}=3.57 . \vartheta=50$. One half of the system is shown. The scale is arbitrary. 


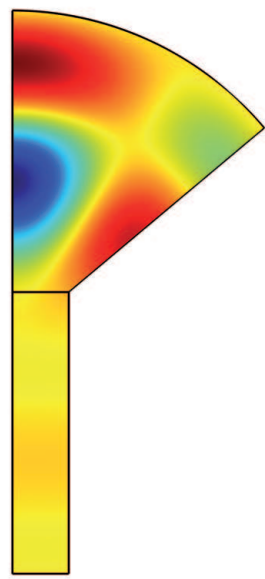

0.1

0.05

0

$-0.05$

$-0.1$

$-0.15$

$-0.2$

Figure 7: Pressure field for a frequency corresponding to a non axi-symmetric mode. $k r_{0}=1.99 . \vartheta=50$. One half of the system is shown. The scale is arbitrary.

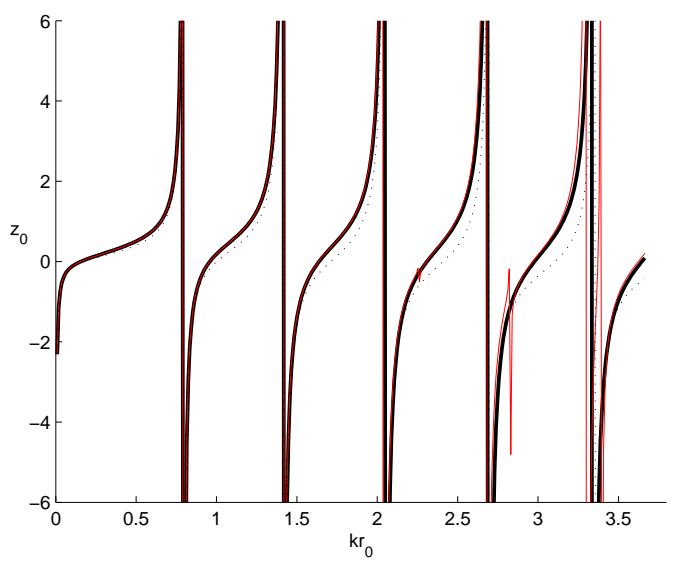

Figure 8: Output impedance $z_{0}$ (imaginary part) of a cylinder terminated in a truncated cone, the latter being terminated into a perfectly rigid spherical cap. $\vartheta=50$. FEM result: grey (red online) line; Formula (17): black line; Formula (20): dotted line. At low frequencies, the FEM results and Eq. (17) are indistinguishable. Above middle frequencies, non-axisymmetric modes appear in the FEM curve. 


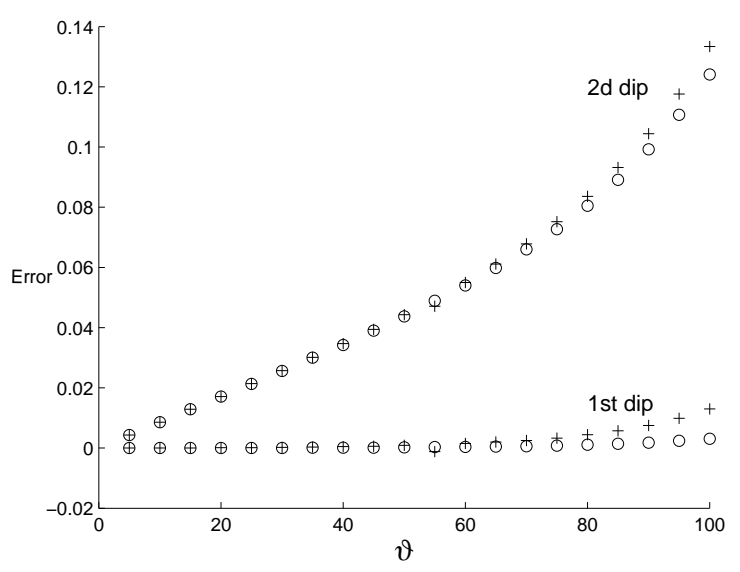

Figure 9: Relative error for the frequencies of the first and second minima of impedance modulus $\left|z_{0}\right|$ ( $\vartheta$ in degrees) ooo Eq. (17), +++ Eq. (20). The termination of the cone is perfectly soft.

4.47 times lower. Therefore a kind of tunneling effect can happen, and cavity modes (i.e., modes of a cone of finite length) can be created (by the change in conicity) without spherical symmetry through evanescent duct modes. It has been checked that for certain frequencies, the spherical symmetry is destroyed in the cone, and nodal lines appear: this is shown in Fig. 7 for the frequency of the first non-axisymmetric mode in Fig. 5, at $k r_{0}=1.99$. This mode shape is similar to those found in the work by Hoersch [11].

Going further in the comparison between numerical results and the formulas, we choose to compute the relative error for the first and second impedance minima (anti-resonances), defined as follows: error $=\left(f_{a} / f_{b}-1\right)$, where $f_{a}$ and $f_{b}$ are the analytical and numerical results, respectively. The relative error for the resonance frequencies is less useful because it is extremely small, and the calculation of an average error on the impedance value over the frequency range would be more difficult to interpret. Figure 9 shows that the error increases monotonically when the angle $\vartheta$ increases. Results for the two formulas (17) and (20) can also be distinguished. The results for an intermediate formula, obtained by using the value of $m$ given by Eq. 25 into Eq. (17) are not shown, because they are very close to those of Eq. (17) with the numerical value of $m$.

Figure 9 shows also some results for obtuse angles, which are discussed in the next section. 


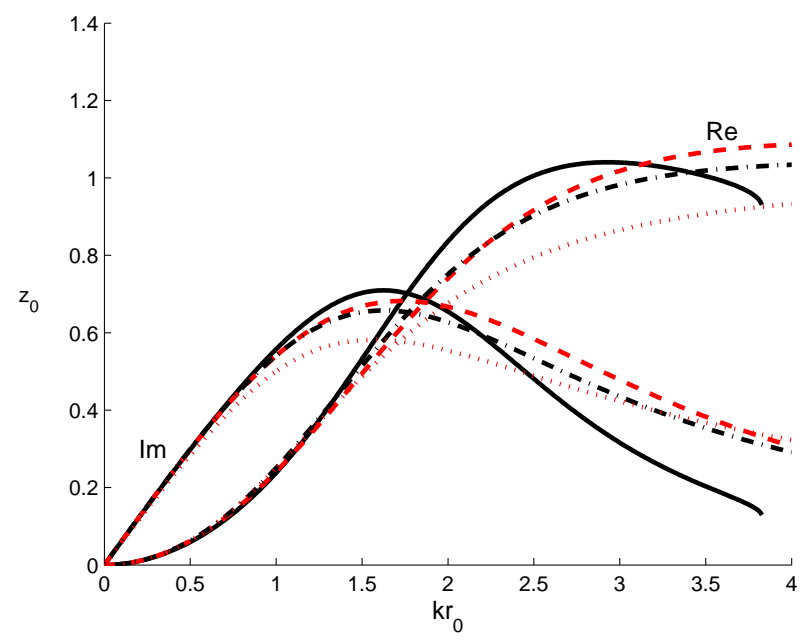

Figure 10: Real and imaginary parts of the radiation impedance $z_{0}$ without flange $(\vartheta=180)$. Black, solid line: exact result [1]; black, dash-dot line, approximate result [9]; grey (red online), dashed line: Eq. (18) for $\alpha=1$; grey (red online), dotted line: Eq. (27).

\section{The case of obtuse angles}

\subsection{Limit of the extension of the previous analysis}

When the angle $\vartheta$ is obtuse, the distance between the plane and spherical surfaces of the matching volume becomes large, and it cannot be regarded as smaller than the wavelength. Therefore it is expected that the results become much less accurate for such angles. However it is observed in Fig. 9 that up to $\vartheta=100^{\circ}$, there is a perfect continuity of the error, and the analytical formulas remain satisfactory. Then, above this value, the error rapidly increases. Therefore another model should be found for a correct description of this case. A recent paper investigated this case using a hybrid FEM [12].

\subsection{Radiation of cylindrical tube without flange}

For the case $\vartheta=\pi$, the results are expected to be very bad, because many coefficients of the formula diverge (this case corresponds to the radiation of a tube without flange, see Ref [1]). The limit of formula (18) is undetermined, and its use is meaningless, because the apex of the cone tends to infinity and therefore the radius $x_{1}$ becomes infinite. 
However another model can be considered, with a sphere having its center at the output of the cylinder, and with a radius $R$. The same calculations as previously described can be made with the following parameters:

$$
\zeta=\frac{\alpha^{2}}{4} ; m_{1}=\frac{\alpha}{4} ; c_{10}=\frac{4}{\alpha^{3}}
$$

where $\alpha=r_{0} / R$. With the knowledge of the added-mass coefficient $m=$ 0.6133, Eq. (18) can be used. Empirically it is found that the best value for $R$ is close to $r_{0}$. We have not yet a clear interpretation of this result. Figure 10 shows the comparison of the exact result of Ref. [1] with that of Eq. (18) for $\alpha=1$ and with the result proposed by Ref. [9]. The accuracy of the two approximate results is similar, but the qualitative conclusion of the present paper remains valid: with the knowledge of the zero frequency added mass, it is possible to deduce a rather satisfactory approximate impedance curve.

Furthermore, the simplified formula (20) leads to the following result:

$$
z_{0}=\frac{7 s / 12+s^{2} / 3}{1+s+s^{2} / 3}
$$

where $7 / 12=0.5833$. A discussion similar to that provided for the infinite flange case can be applied, but this formula is less satisfactory than Eq. 22 (see Fig. 10).

\section{Small angles; discussion of previous results of the literature for the case of an infinite cone}

The present section aims to discuss the results of Chester [3] and Martin [4] concerning the case of an infinite cone. The comparison will be done for the modulus of the reflection coefficient of planar waves in the cylindrical duct (for brevity we do not discuss the behavior of the argument).

Chester gave an analytical formula, obtained with the following hypothesis: the expansion in modes of the cylindrical duct can be extended inside the matching volume (notice that this implies that the rigid walls of the cylinder are elongated). Eqs. (15) and (4) of Chester's paper give the value of the impedance $z_{0}$. Numerical coefficients are given by a table. We do not reproduce the data here, but Fig. 11 shows the result, and compares it to Formulas (17) and (20), with $R_{f}=\left(z_{0}-1\right) /\left(z_{0}+1\right)$, for 3 values of $\vartheta$ and with the exact result for $\vartheta=90$. Chester's formula appears to be useful only 


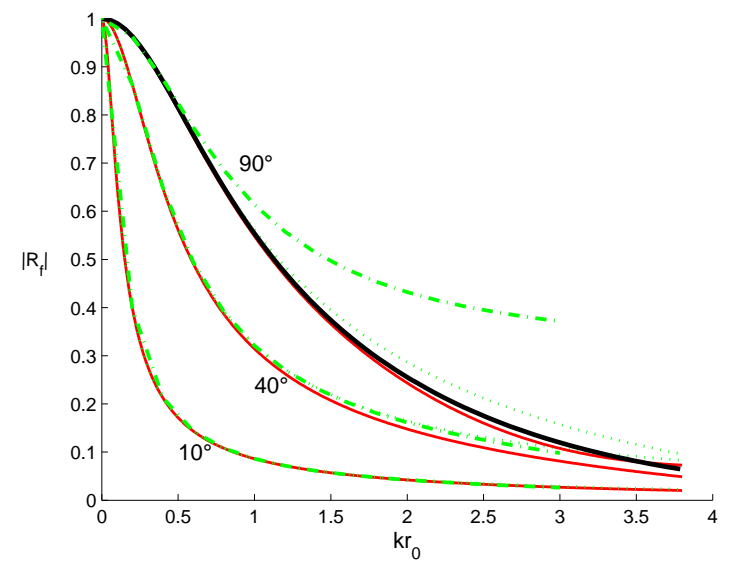

Figure 11: Modulus of the reflection coefficient at the output of the cylinder for three angles: 10, 40 and 90 degrees. Comparison of Martin's formula (dotted line, green online) and Chester's formula (dash-dot line, green online) with Eq. (17) (solid line, red online). The black, thick line is the exact result for 90 degrees.

at rather small angles. It can be noticed that the author himself mentioned that for smaller angles the result is very close to that of a simplified formula, which it turns out is $z_{0}=1 / y_{1}$ (with the addition of a phase shift).

In Ref. [4], Martin gave another approximate formula. The method was the analytical application of the Green theorem, and the expected validity is for small angles and low frequencies. The formula can be written in the form:

$$
R_{f}=\frac{\sin ^{2} \vartheta-4 \zeta[\exp (-s \sin (\vartheta) /(2 \zeta))-1]}{-\sin ^{2} \vartheta+4 \zeta s^{2}} .
$$

Figure 11 shows that it is an excellent approximation for small angles, and it is less satisfactory for wide angles.

Furthermore Martin discussed simplified formulas for small angles and low frequencies. He gave an expansion with respect to frequency, limited to the first order of the frequency. This yields $R_{f}=-1+2 s \csc \vartheta$, and a negative real part of the impedance $z_{0}$. However if the orders of magnitude of $\vartheta$ and $s$ are considered to be similar, the following formula is found:

$$
R_{f}=-\frac{\vartheta}{\vartheta+2 s} .
$$

This result is the same by using either Formula (20) or Formula (28), the missing terms being of the $3 r d$ order $\left(s \vartheta^{2}, \vartheta s^{2}\right)$. This corresponds to Eq. (6) 
for small angles, therefore to the assumption that both the mass $\left(m-m_{1}\right)$ and the matching volume can be ignored. This approximation is consistent with the approximation of the horn equation (often called "Webster equation") written in spherical waves (see [7, 13]), which leads to an accuracy much higher than that using plane waves [14].

\section{Conclusion}

The main interest of the general analysis presented in the paper is the possibility to derive a knowledge of a complete impedance curve from the knowledge of the geometry and one supplementary parameter only: the zero frequency added mass. Formula (17) together with the fit formula (25) gives very satisfactory results when compared to numerical results for finite length cones and acute angles.

The case of obtuse angles seems to be more difficult, even if the results for slightly obtuse angles are not bad. The limitation is not only relative to angles, but also to frequencies: this is not surprising, and anyway in practice, for imperfect cylindrical tubes, the appearance of higher order propagating modes occurs near $k r_{0}=1.8$.

The results for the standard case of the radiation of a tube without flange or with an infinite flange compare favorably with the fit formulas obtained by optimization in Ref. [9].

It is remarkable that approximate formulas can take various forms (see Refs. [3, 4, 9]). Our formulation derives from an electrical circuit. Would it be possible to improve it? Three new parameters would be added if the third-order terms in Eq. (4) are included. This would likely be possible, but in practice, situations at higher frequencies with one propagating mode only are rare.

Finally, it is probable that the use of the general formulation (4) could be used also for other problems, such as the matching of truncated cones with different angles. The treatment of radiation of tubes with flanges of finite thickness [15, 16] could also be an interesting problem to investigate. Furthermore the knowledge of various cases of the cone terminal impedance on surface $S_{2}$ would be an important subject, in order to extend the utility of the general analytical presented in this paper. 


\section{Appendix A: Formula for a junction of two waveguides at low frequencies}

We consider the junction of two waveguides, with rigid walls. Fig. 1 shows the example studied. In each of them, a surface normal to the guide axis is chosen (plane if the guide is cylindrical, spherical if it is conical). The surface areas are $S_{0}$ and $S_{1}$. In each guide, the choice of the abscissa of these surfaces is arbitrary, but it is useful to choose the junction cavity as small as possible. In each guide, several duct modes exist. In the junction cavity, the following classical integral equation can be written in the frequency domain:

$$
p(\mathbf{r})=j \omega \rho \int_{S} G\left(\mathbf{r}, \mathbf{r}^{\prime}\right) v\left(\mathbf{r}^{\prime}\right) d S^{\prime},
$$

where $S$ is the surface of the cavity and $G\left(\mathbf{r}, \mathbf{r}^{\prime}\right)$ the Green's function for the cavity with rigid walls (Neumann boundary conditions). $p$ and $v$ are the acoustic pressure and normal velocity in the cavity, $\mathbf{r}$ and $\mathbf{r}^{\prime}$ the coordinate vector of two points in the cavity. In the present case $S=S_{0}+S_{1}$. For other choices of the surfaces $S_{i}(i=0$ or 1$)$, a part of $S$ can be a rigid wall, with a vanishing normal velocity. In Guide $i$, the eigenmodes are denoted $\psi_{i p q}(\mathbf{w})$, where $\mathbf{w}$ is the $2 \mathrm{D}$ vector on the surface. A column vector $\boldsymbol{\psi}_{i}$ is built, by ordering the (double infinity of) modes of Guide $i$ in order by cutoff frequency. Orthogonality can be written as a matrix relationship:

$$
S_{i}^{-1} \int_{S_{i}} \boldsymbol{\psi}_{i}{ }^{t} \boldsymbol{\psi}_{i} d S=\mathbf{1}
$$

where $\mathbf{1}$ is the identity matrix. Then, two vectors, $\mathbf{P}_{i}$ and $\mathbf{U}_{i}$, are built with the coefficients for the expansion of the pressure and normal velocity fields on the surface $S_{i}$, as follows:

$$
p\left(\mathbf{w}, z_{i}\right)={ }^{t} \boldsymbol{\psi}_{i}(\mathbf{w}) \mathbf{P}_{i} \text { and } v\left(\mathbf{w}, z_{i}\right)=S_{i}^{-1}{ }^{t} \boldsymbol{\psi}_{i}(\mathbf{w}) \mathbf{U}_{i},
$$

$z_{i}$ is the axial coordinate on the surface $S_{i}$ in Guide $i$. Because of the Neumann condition on the walls, one of the modes is the uniform mode (also called fundamental mode, i.e., planar or spherical), independent of the vector $\mathbf{w}$. With the expansion (A3), the integral equation can be re-written in the form of a matrix relationship:

$$
\left(\begin{array}{c|c}
\mathbf{P}_{0} \\
\hline \mathbf{P}_{1}
\end{array}\right)=\left(\begin{array}{c|c}
\mathbf{Z}_{00} & \mathbf{Z}_{01} \\
\hline \mathbf{Z}_{10} & \mathbf{Z}_{11}
\end{array}\right)\left(\begin{array}{c}
\mathbf{U}_{0} \\
\hline \mathbf{U}_{1}
\end{array}\right)
$$

where the matrices $\mathbf{Z}_{i j}$ are:

$$
\mathbf{Z}_{i j}=\frac{j \omega \rho}{S_{0} S_{1}} \int_{S_{i}} \int_{S_{j}} \boldsymbol{\psi}_{i} G\left(\mathbf{r}, \mathbf{r}^{\prime}\right)^{t} \boldsymbol{\psi}_{j} d S_{i} d S_{i}^{\prime} .
$$


The dimension of these four matrices is doubly infinite. It is now possible to write Eq. (A4) in the form of a super-matrix impedance:

$$
\mathbf{P}=\mathbf{Z U}
$$

The first step of the proof is achieved. The second step consists of the separation of propagating and evanescent modes. The vector $\mathbf{P}$ is re-ordered, writing first the vector $\mathbf{p}$ of the propagating modes (2 elements, because at low frequencies, only the fundamental modes propagate), then the vector $\mathbf{P}^{\prime}$ of the evanescent modes (similarly for $\mathbf{U}$ ). Therefore the matrix $\mathbf{Z}$ can be partitioned in four parts, as follows:

$$
\left(\begin{array}{c}
\mathbf{p} \\
\hline \mathbf{P}^{\prime}
\end{array}\right)=\left(\begin{array}{c|c}
\mathbf{z} & { }^{t} \mathbf{z}^{\prime} \\
\hline \mathbf{z}^{\prime} & \mathbf{Z}^{\prime}
\end{array}\right)\left(\begin{array}{c}
\mathbf{u} \\
\hline \mathbf{U}^{\prime}
\end{array}\right)
$$

$\mathbf{z}$ is the impedance matrix (of order 2) restricted to the propagating modes in the two guides, $\mathbf{z}^{\prime}$ is the impedance matrix relating one propagating mode and one evanescent mode, ${ }^{t} \mathbf{z}^{\prime}$ is the transpose of $\mathbf{z}^{\prime}$ (because of reciprocity, the matrix $\mathbf{Z}$ is symmetrical), and $\mathbf{Z}^{\prime}$ is the matrix $\mathbf{Z}$ restricted to the evanescent modes. If the guides are long enough, their lengths are infinite for the evanescent modes, and the impedance of each of them is its characteristic impedance $\mathbf{Z}_{c}^{\prime}$, which is a diagonal matrix:

$$
\mathbf{P}^{\prime}=-\mathbf{Z}_{c}^{\prime} \mathbf{U}^{\prime}
$$

Finally, using Eqs. (A7) and (A8), the impedance matrix relationship for the propagating modes is found to be:

$$
\mathbf{p}=\left[\mathbf{z}-{ }^{t} \mathbf{z}^{\prime}\left(\mathbf{Z}^{\prime}+\mathbf{Z}_{c}^{\prime}\right)^{-1} \mathbf{z}^{\prime}\right] \mathbf{u}
$$

In order to calculate the partition, we write:

$$
\psi_{i}=\left(\frac{1}{\psi_{i}^{\prime}}\right)
$$

Orthogonality of the uniform modes with any other mode implies:

$$
\int_{S_{i}} \boldsymbol{\psi}_{i}^{\prime} d S=\mathbf{0}
$$

(only the fundamental mode contributes to the average quantities). We are interested in the series expansion of the result with respect to frequency. At low frequencies, the characteristic impedance of the evanescent modes, which is purely imaginary, is proportional to $j \omega \rho$ (this comes from the Euler 
equation). The 3D modal expansion of the Green's function of a closed cavity is classically written as follows:

$$
G\left(\mathbf{r}, \mathbf{r}^{\prime}\right)=\frac{1}{V} \sum_{m n p} \frac{\phi_{m n p}(\mathbf{r}) \phi_{m n p}\left(\mathbf{r}^{\prime}\right)}{k_{m n p}^{2}-k^{2}}
$$

where $\boldsymbol{\phi}_{m n p}(\mathbf{r})$ are the orthonormal modes in the cavity. Because the cavity is closed with Neumann boundary conditions, a uniform, fundamental 3D mode exists with $k_{000}=0$, and the following expression can be derived at the zeroth order in $\omega$ :

$$
G\left(\mathbf{r}, \mathbf{r}^{\prime}\right)=-c^{2}\left(\omega^{2} V\right)^{-1}+G_{0}\left(\mathbf{r}, \mathbf{r}^{\prime}\right)+O\left(\omega^{2}\right),
$$

$G_{0}\left(\mathbf{r}, \mathbf{r}^{\prime}\right)$ is the sum of the term corresponding to the non-uniform modes, which is independent of frequency. The missing term is of order $\omega^{2}$. Analyzing the frequency dependence of the terms in Eq. (A9), it appears that at low frequencies:

- the matrix $\mathbf{z}$ can be divided in two terms as shown in Eq. (4).

- the matrices $\mathbf{z}^{\prime}$ and $\mathbf{Z}^{\prime}$ are proportional to $j \omega \rho$ : because of Eq. (A11), there is no term inversely proportional to $j \omega$.

Finally the matrix ${ }^{t} \mathbf{Z}^{\prime}\left(\mathbf{Z}^{\prime}+\mathbf{Z}_{c}^{\prime}\right)^{-1} \mathbf{z}^{\prime}$ is proportional to $j \omega \rho$, and Eq. A9 yields the shape of Eq. (4).

We remark that when the junction cavity has a zero volume, Eq. (4) has to be replaced by $U_{0}=U_{1}$ (in order for the pressure to be finite, the sum of the flow rates vanish), and

$$
P_{0}-P_{1}=j \omega\left(\begin{array}{ll}
M_{00}-M_{10} & \left.M_{01}-M_{11}\right)
\end{array}\right)\left(\begin{array}{c}
U_{0} \\
-U_{1}
\end{array}\right)
$$

Formula $(\mathrm{A} 9 \mathrm{p}$ can be generalized to an arbitrary number of guides as well as an arbitrary number of propagating modes. Equation (4) can be generalized to an arbitrary number of guides where only the fundamental mode propagates, as well as Eq. A14 for the case $V=0$. The latter case, without compressibility effect, has been treated in many papers of the literature for the problems of 2- , 3- or 4-guide junction using either the mode-matching method, or the conformal mapping (see, e.g., [16 21]). The matched asymptotic expansion was also used in order to find the shape of the impedance matrix (or of the equivalent circuit (see, e.g., [19,22]).

\section{Acknowledgements}


This work was financed by the research project CAGIMA supported by Agence Nationale de la Recherche. We also acknowledge the support of the Natural Sciences and Engineering Research Council of Canada and the Centre for Interdisciplinary Research in Music Media and Technology at McGill University.

\section{References}

[1] H. Levine and J. Schwinger, On the radiation of sound from an unflanged circular pipe. Phys. Rev. 73 (1948), 383-406.

[2] A.N.Norris, I.C. Sheng, Acoustic radiation from a circular pipe with an infinite flange. J. Sound Vib. 135 (1989) 85-93.

[3] W. Chester, The acoustic impedance of a semi-infinite tube fitted with a conical flange: Part II. J. Sound Vib. 116 (1987) 371-377.

[4] P.A. Martin, The horn-feed problem: sound waves in a tube joined to a cone, and related problems. J. Eng. Math. 71 (2011) 291-304.

[5] J. Kergomard, A. Khettabi, A. Garcia, General formulation of waveguide junction at low frequencies, C.R. Acad. Sci., Paris, t. 319, II, (1994), 887-892.

[6] R.Caussé, J.Kergomard, X.Lurton, Input impedance of brass musical instruments: comparison between experiment and numerical models. J. Acoust. Soc. Am. 75 (1984) 241-254.

[7] A.H. Benade and E.V. Jansson. On plane and spherical waves in horns with nonuniform are. I. Theory of Radiation, Resonance Frequencies, and Mode Conversion. Acustica 31 (1974) 79-98.

[8] L. V. King, On the electrical and acoustic conductivities of cylindrical tubes bounded by infinite flanges. Phil. Mag. 21 (1936),128-144.

[9] F. Silva, Guillemain, P., Kergomard, J., Mallaroni, B., Norris, A., Approximation of the acoustic radiation impedance of a cylindrical pipe. J. Sound Vib., 322 (2009), 255-263.

[10] L.C. Tyte, Some notes on end-corrections, Phil. Mag. 7, 30 (1940), 173184. 
[11] V.A. Hoersch, Non-radial harmonic vibrations within a conical horn, Phys. Rev. 25 (1925), 218-224.

[12] W. Duan and R. Kirby, A hybrid finite element approach to modeling sound radiation from circular and rectangular ducts, J. Acoust. Soc. Am., 131 (2012), 3638-3649.

[13] T. Hélie. Unidimensional models of acoustic propagation in axisymmetric waveguides. J. Acoust. Soc. Am., 114 (2003) 2633-2647.

[14] T. Hélie, T. Hézard, R. Mignot, D. Matignon, One-Dimensional Acoustic Models of Horns and Comparison with Measurements. Acta Acustica united with Acustica, 99 (2013) 960 - 974.

[15] Y. Ando, On the sound radiation from semi-infinite circular pipe of certain wall thickness. Acustica 22 (1969) 219-225.

[16] J. P. Dalmont, C. J. Nederveen, N. Joly, Radiation impedance of tubes with different flanges: Numerical and experimental investigations, J. Sound Vib. 244 (2001) 505-534.

[17] Miles, J.W., The Equivalent Circuit for a Bifurcated Cylindrical Tube. J. Acoust. Soc. Am. 19 (1947) 579-584.

[18] Miles J.W., On the Change of Cross Section and Bifurcation of a Cylindrical Tube, J. Acoust. Soc. Am. 22 (1950) 59 - 60.

[19] J.C. Bruggeman, The propagation of low-frequency sound in a twodimensional duct system with $\mathrm{T}$ joints and right angle bends: Theory and experiment. J. Acoust. Soc. Am. 82 (1987) 1045-1051.

[20] J. Kergomard, A. Khettabi, X. Mouton, Propagation of acoustic waves in two waveguides coupled by perforations. I. Theory. Acta Acustica 2 (1994) 1-16.

[21] V. Dubos, J. Kergomard, A. Khettabi, J.P. Dalmont, D. Keefe, C.J. Nederveen, Theory of Sound Propagation in a Duct with a Branched Tube Using Modal Decomposition, Acustica united with Acustica, 85 (1999) 153- 169.

[22] M.B. Lesser and J.A. Lewis, Applications of Matched Asymptotic Expansion Methods to Acoustics. I. The Webster Horn Equation and the Stepped Duct. J. Acoust. Soc. Am. 51 (1972) 1664-1669. 